\title{
An Approach to E-marketplace Automation
}

\author{
Bojan MILIĆ, Bojan ROSI, Roman GUMZEJ
}

\begin{abstract}
E-marketplaces have become an essential part of e-commerce. In our research a decentralised agent-based e-marketplace platform was devised. The goal of our research is to improve overall supply chain service quality by allowing companies' agents to evaluate the service quality of their partners through the history of their transactions. Consequently, since more informed decisions are taking place continuously and autonomously, supply chain service quality is being improved along the whole supply chain. In the article a service quality evaluation model of a supply chain is empirically evaluated.
\end{abstract}

Keywords: e-commerce; e-marketplace; agent-based systems; supply chain management; service quality

\section{INTRODUCTION}

E-marketplaces have become an essential part of ecommerce. The centralised web-site based approach is being successfully exploited by companies not being connected by long-term contracts and direct e-business relations. Multiple such e-marketplaces have been established mostly being used by companies of a common industry to help them manage their supply chains.

The purpose of this research is to devise a decentralised agent-based e-marketplace. As companies arise, they are assigned agents that represent them in the marketplace and are registered with a yellow-page service corresponding with their line of business in general and their products in particular. This service, being used by all companies' agents on the e-marketplace, manages the routing of their inquiries for specific goods. As companies arise and vanish they are registered and de-registered from this service. Once the proposals have arrived from supplier companies' agents, a selection and ordering procedure is conducted between a customer and its supplier agents until the transaction is completed.

In the article an analytical service quality evaluation model of a supply chain is empirically evaluated synthetically, by an analogous agent based supply chain model within our e-marketplace, and analytically, by statistical analysis of the decisions made based on the same transaction data. Based on the results we may conclude that in contrast to centralised e-marketplace platforms, the agent-based approach is distributed and dynamic, and hence corresponds more closely with the supply chain's natural behaviour. In addition, by utilising service qualityoriented decision support and making increasingly informed decisions the service quality within the entire supply chain of any contributing company is gradually improved up- and downstream.

\subsection{Motivation}

With increasing competition in the global market, organisations are starting to realise that it is not enough to merely improve their efficiency internally, but it is also necessary to consider the competitiveness and efficiency of their whole supply chain. Performance measurement and optimisation play a vital role in improving the competitiveness of supply chains [1]. Moreover, with the rapidly developing world economy and global marketplaces, there has been a drastic increase in the pressure on organisations to find new ways to create and deliver value to customers through supply chain management. There has been a growing recognition of the importance of building relationships with customers for improvements in profitability, as well as serviceability and reduced costs throughout the supply chain [2].

Different authors [3] emphasise the importance of delivering superior total value to the customer in terms of promptness, cost, quality and flexibility rather than by focusing solely on promptness and cost. According to [4] service quality has been a major area of attraction for practitioners and researchers. Its proven relationship with improving business performance by lowering costs, increasing customer satisfaction, achieving customer loyalty and increasing profitability has further motivated both researchers and practitioners to explore this area.

The biggest problem that modern organisations face today is their inability to quickly adapt to changes dictated by fast-evolving supply chains. More specifically, organisations currently rely on various mechanisms to perform quality assessment of their partners and adapt their decision-making processes to improve their business performance. Hence, numerous research studies have dealt with the subject of service quality in supply chains and a consensus has been reached concerning a strong relationship among service quality and supply chain performance [5].

To achieve the desired global optimisation throughout the entire supply chain, one must employ proper mechanisms to measure, monitor and control service quality through an interorganisational assessment system [6]. Such systems are sensibly implemented to identify opportunities for improved supply chain efficiency and competitiveness, to help understand how companies operating in supply chains affect each other's performance, to support the supply chain in satisfying consumer requirements and to assess the result of an implemented initiative [7]. As stated by [8] for collaborative networks, supply chains also have the need for decentralised operations where partners conduct their planning autonomously and only exchange a limited amount of information. A frequently proposed solution concept for this purpose are auction mechanisms, and more specifically multi-agent systems.

The goal of this research is to establish an agent-based autonomous e-marketplace framework to improve overall 
supply chain service quality. Any e-commerce transaction involving a pair of supply chain nodes, fulfilling the customer-supplier relationship, is automatically integrated into a service-quality-oriented decision model, where the current transaction data are supplemented with historic data on previous transactions between the respective partners, with the goal of best meeting customer's needs. Consequently, since more informed decision making is taking place autonomously along the whole supply chain, its service quality is being improved up- and downstream. In contrast to centralised e-marketplace platforms, the agent-based approach is distributed and dynamic, and hence corresponds with supply chains' natural behaviour.

This research relies on the holistic approach to service quality assessment defined in $[9,10]$. The main reasons for employing a holistic approach are in consequence of the otherwise identified absence of connection with a strategy, lack of system thinking, in which a supply chain must be viewed as a whole entity, lack of balanced approach integrating financial and non-financial measures, as well as loss of supply chain context, thus encouraging local optimisation. Based on the service quality assessment model a novel multi-agent based collaboration framework for interorganisational (supply chain) cooperation and service quality assessment is introduced that can perform supply chain management operations synchronously, according to the mentioned goals.

The structure of this article is as follows. First, contemporary multi-agent systems in supply chains are reviewed. In the sequel, the proposed multi-agent supply chain management framework for improving overall service quality within supply chain(s) is presented. To supplement our framework, an e-marketplace Web 2.0 ontology has been defined. It is meant for conceptualising and managing supply chain nodes' collaboration and knowledge and is laid out next. To analyse and evaluate our approach, the results of a simulation study on our model are presented and statistically assessed. In the conclusion the main outcomes of our research are discussed.

\subsection{Multi-Agent Systems in Supply Chains}

According to [11] automation of supply chain management systems has long been a principal use of both academia and industry. Generally, two approaches of service transformation in the digital era can be observed for efficient, service-quality-oriented supply chain management:

(1) Centralised e-marketplace web portals, combining demand and supply in a temporally and spatially shared repository $[12,13]$ and

(2) Decentralised, agent-based e-marketplaces with a yellow-page dictionary service and distributed network of temporally and spatially distributed agents of supply chain partners [11, 14-25].

Each approach focuses on enriching some aspects of traditional supply chain management information systems. The centralised e-marketplace approach facilitates interoperability amongst supply chain participants, regardless of their information system diversity. On the other hand, the participants are left with a limited degree of autonomy, and without the capacity to adapt in a dynamic way to changing real-world situations. In case such situations arise, intelligent agents can provide the participating systems with a high degree of autonomy and dynamicity [11].

The academic literature shows that many authors have worked on enhancing their supply chain operations using multi-agent technologies. Ref. [26] have proposed a framework for the design of a multi-agent-based decision support system to manage disruptions and mitigation of risks in manufacturing supply chains. Ref. [16] has proposed a model that consists of seven agents that are working together to maintain supply, manufacture, inventory management and distribution. Each agent performs a specific function of the organisation and shares the information with other agents. Ref. [17] presented an agent-based approach for supply chain event management problems, which can perform autonomous corrective control actions to minimise the effect of deviations in the plan that is currently being executed. Ref. [18] presented an agent-based framework for configuration of agile supply chains to demonstrate application of agent technology for supply chain configuration based on a formal ontology that encodes the manufacturing capabilities of manufacturing suppliers. Ref. [21] worked on ontology-based negotiation knowledge using agent technologies and showed that by using this method negotiation behaviours would be more adaptive to various negotiation environments. Ref. [22] focused on collaboration of demand, production and replenishment planning along a supply chain and proposed a multi-tier, negotiation-based mechanism supported by a multi-agent system. Ref. [23] presented a concept and application of a hybrid multi-agent approach to modelling and optimisation of supply chain problems. They integrated mathematical programming and constraint logic programming using agent technology. Ref. [19] proposed SCOPE, a multiagent modelling and simulation platform specifically designed to emulate and study complex supply chain systems. Ref. [24] used a case study to confirm that environmental uncertainty can be automatically reduced to a win-win mode demonstrating the self-adaptive property of the multi-agent supply chain systems. Ref. [25] proposed a multi-agent architecture which helps medium size enterprises to take an appropriate decision to mitigate uncertainty in the supply chain.

While many authors have worked on enhancing their supply chain operations using multi-agent technologies, none have focused on supply chain automation using a service quality assessment model, as defined in $[9,10]$. Considering the service quality criteria, by representing a supply chain as a multi-agent system and making supply chain operation decisions according to this service quality assessment model, up to $50 \%$ better informed decisions could be made, resulting in higher customer satisfaction downstream and hence better overall service quality of the supply chain.

In the sequel, the proposed multi-agent based collaboration framework with interorganisational (supply chain) service quality assessment is presented. 


\section{MULTI-AGENT FRAMEWORK WITH SERVICE QUALITY ASSESSMENT FOR COLLABORATION WITHIN SUPPLY CHAINS}

According to [27] agent architectures are fundamental mechanisms underlying the autonomous components that support effective behaviour in real-world, dynamic and open environments. Agents are considered one of the most important paradigms that on one hand may improve current methods for conceptualising, designing and implementing software systems and on the other may represent a solution to the legacy software integration and automation problems.

In artificial intelligence, an intelligent agent is an autonomous entity which observes through sensors and acts upon an environment using actuators (i.e. it is an agent) and directs its activity towards achieving goals (i.e. it is "rational", as defined in economics [28]. Intelligent agents may also learn or use knowledge to achieve their goals.

To apply the decentralised agent-based approach to supply chain management, any pair of supply chain nodes shall be autonomously integrated into both-the information and material flows-with the goal of meeting supply chain nodes' service quality requirements. By their behaviour they are classified as model-based learning agents.

In the following section, the proposed framework is presented in detail. First, the service quality assessment model used when performing supply chain operation decisions is presented. Then the agent knowledge sharing Web 2.0 ontology is laid out. Finally, the agent communication and behaviour models are presented.

\subsection{Service Quality Assessment Model}

Overall, service quality assessment requires performance indicators that pertain to the service quality among supply chain partners (nodes) of a supply chain. To track service quality along supply chains, probes (defined by $Q$ ) are placed between every pair of interlinked nodes. Since some of the performance indicators defined in [9] are qualitative, they are not suitable for automated service quality evaluation. Hence, only the quantitative service quality indicators shall form the basis of evaluation. We introduce the $Q$ as a 4-tuple of service quality indicators $(C, T, D, P)$ representing service quality on every link between a chosen pair of supply chain nodes. All mentioned service quality indicators were assigned an equation that best expresses their interrelations with the node's inputs (e.g. burden/load) as well as with other service quality criteria. Finally, overall service quality $(Q)$ has been expressed as a normalised sum of these criteria.

Correctness represents the proportion of correctly completed transactions:

$$
C=\frac{c}{d}
$$

where $c$ represents the number of correctly fulfilled orders and $d$ the total number of orders.
Timeliness represents the proportion of timely completed transactions:

$$
T=\frac{t}{d}
$$

where $t$ represents the number of on-time fulfilled orders and $d$ the total number of orders. Timely fulfilled orders are those whose processing time $\left(t_{\text {out }}-t_{\text {in }}\right)$ is less than the time specified for delivery.

Dependability represents the sum of availability $(A)$ and reliability $(R)$, defined in the sequel:

$D=\frac{A+R}{2}$

Since the individual summands in (3) are in the $(0,1)$ range, in order to normalise the result, it is divided by 2 .

Availability represents the proportion of time a node is in a functioning condition (available to its customer's orders):

$$
A=1-\frac{M T T R}{M T T F},
$$

where $M T T F$ represents its average Mean-Time-To-Failure and MTTR its average Mean-Time-to-Repair (e.g. maintenance operations or other exceptional situations, which prevent a node from responding immediately to an incoming request).

Reliability can be expressed as the opposite of a node's fault rate:

$R=1-F$

where $F$ represents the ratio of failures to fulfil requests (e.g. due to out-of-stock situations). Reliability is considered the ratio/percentage of cases when the observed process in a supply chain did not fail to complete.

Price is usually calculated based on the cost of processing items at each node, namely:

$P=\frac{C M+P Q \times c p+(T S+T P) \times c t}{P Q}$,

where $C M$ represents cost of material, $P Q$ processing quantity, $c p$ cost per item (product/service cost), TS time to process (service time) and $T P$ time to prepare the process with appropriate ct cost of an item's processing time. In order to calculate the cost of a single item, the price should be divided by the processing quantity $(P Q)$.

The price of an item $(P)$ should be divided by the highest price $(\max [P])$ to render the price indicator for our service quality evaluation $\left(Q_{\mathrm{p}}\right)$, namely:

$Q_{\mathrm{p}}=1-\frac{P}{\max [P]}$,

Finally, the overall service quality can be calculated as a normalised sum of these indicators: 


$$
Q=\frac{C+T+D+Q_{\mathrm{p}}}{4},
$$

Since the individual summands are all in the $(0.1)$ range, in order to normalise the result, it is divided by 4 . While finetuning the model, we might decide to ponder the individual summands, as indicated in [9], however for the purpose of this initial study this could be misleading and result in false conclusions about the service quality evaluation model.

\subsection{Agent Knowledge Sharing}

Each multi-agent system needs a mechanism for knowledge sharing. Agent communication protocols may be utilised to access this knowledge and to establish sensible relations among supply chain nodes as well as to implement appropriate behavioural models.
According to [29] ontologies were developed in artificial intelligence (AI) to facilitate knowledge sharing and reuse. They enable a shared and common understanding of a defined domain that can be communicated between people and application systems. In our case, it is meant for conceptualising and managing supply chain knowledge, agent communication protocols by which this knowledge is shared between supply chain nodes and autonomous agent behaviour models.

Our ontology establishes the basic vocabulary of terms which are relevant for e-commerce. Hence, we have named it e-marketplace (see Fig. 1). The main concepts it introduces are associated with supply chain nodes, represented by agents and transactions that are exchanged among them. It was created using the Stanford's Protégé tool.

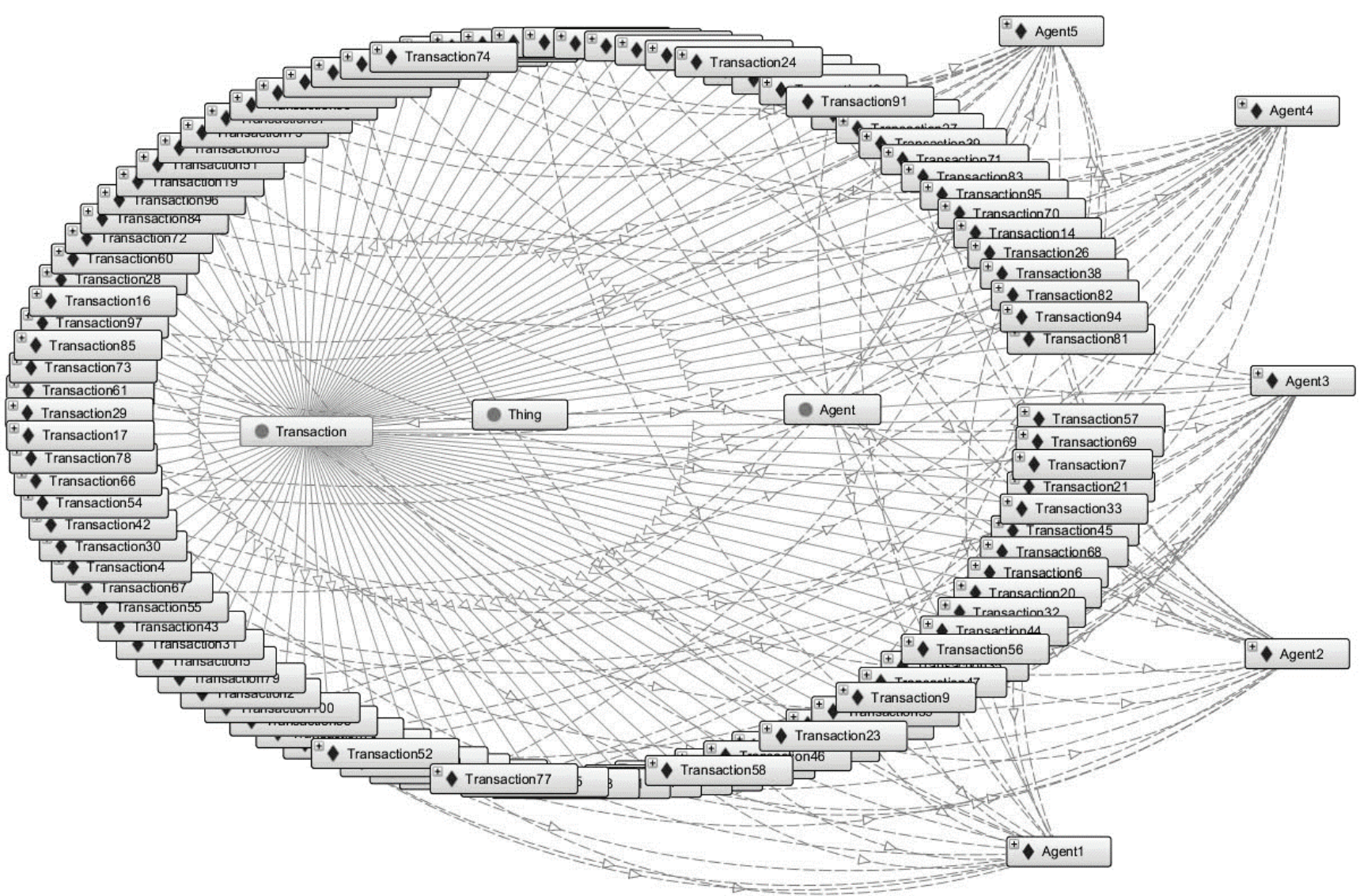

Figure $1 \mathrm{E}$-marketplace supply chain ontology

Class Transaction represents business transactions among individual nodes of our supply chain. These business transactions are assigned to class Agent, which can represent different types of supply chain nodes (supplier, distributor, customer, etc.). Classes Transaction and Agent are disjoint, which means that any individual node (object) cannot be an instance of more than one of these two classes. To match our statistical evaluation, we have defined 100 transactions and split transaction objects evenly among our 5 supplier agent objects (Agent 1 to 5), who are members of our customer agent's (Agent) supply chain.

In the sequel agent communication protocols that are needed to perform supply chain operations and utilise knowledge shared by the e-marketplace ontology are presented.

\subsection{Agent Communication}

A key component in any multi-agent based system is communication. According to [27], agents need to be able to communicate with users, system resources and with each other to collaborate and negotiate. In particular, agents interact with each other by using agent communication language (ACL). According to FIPA (the Foundation for Intelligent, Physical Agents) each agent has its own characterisation, which represents its behaviour. Agents' messages represent actions or communicative acts-also 
known as speech acts of performatives. It is stated in the FIPA standards that, to be fully compliant, agents must be able to receive any legal FIPA-ACL message and at the very least respond with a not-understood message, in case the processing of the message does not render a meaningful result. FIPA-ACL messages contain a set of one or more message parameters. Precisely which parameters are needed for effective agent communication varies according to situation [27].

Our autonomous agents communicate over HTTP protocol and are based on the FIPA Contract Net Protocol [30], which allows one agent, the Initiator, to have some tasks performed by one or more other agents (Participants) and further optimisation of a function that characterises the task. For a given task, any number of Participants may respond with a proposal; the rest must refuse. The initiator then continues negotiations with the participants that proposed.

In Fig. 2 the communication among five agents, representing suppliers, and one agent representing a customer is shown, according to the FIPA Contract Net Protocol for our e-commerce supply chain ontology. First, the customer object reads the data on the potential supplier objects. Then it contacts them with a call for proposal
(CFP) message indicating transaction data. The supplier objects answer with their proposals (PROPOSE) according to their transaction data. After comparing the incoming proposals and selecting the most appropriate one, the customer object informs the owner of the selected transaction that it accepts its proposal (ACCEPTPROPOSAL) and the rest that it rejects their proposals (REJECT-PROPOSAL). The selected supplier object fulfils the order of the customer object by informing it about this fact, which results in appropriate fulfilment actions.

All agents are generic until they are enlisted as members of a supply chain. When agents are registered in yellow page service, their specific role (customer, supplier, customer and supplier) is indicated. Every pair of supply chain nodes that interact with each other can be observed as a customer-supplier relation. A customer orders material, a service or a finished product (item) from its suppliers based on their respective price and service quality.

When an agent of a type supplier is being registered in yellow page services, the query is made to the emarketplace ontology for that agent (process shown in Fig. $3)$.

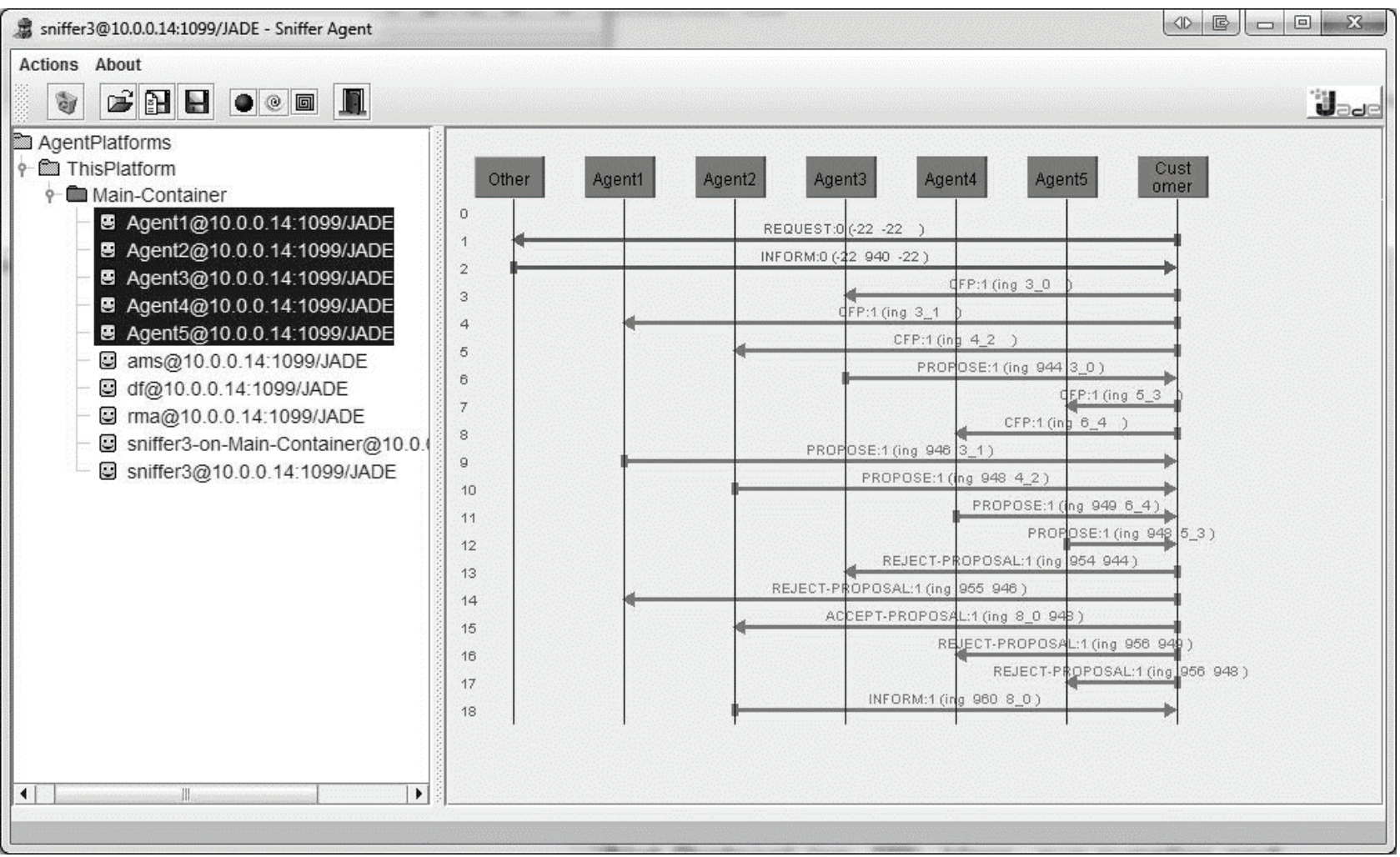

Figure 2 E-marketplace supply chain communication protocol

Based on that query, agent-related indicators are saved into a SupplierContentMessage object, which consists of the following information:

- Correctness: average value of the Correctness indicator for all business transactions made in the history of participation in the supply chain;

- Timeliness: average value of the Timeliness indicator for all business transactions made in the history of participation in the supply chain;
- Dependability: average value of the Dependability indicator for all business transactions made in the history of participation in the supply chain;

- Offered price $(P)$ : value of the current offered price for specific item;

- Agent name: unique identifier of the supplier. 

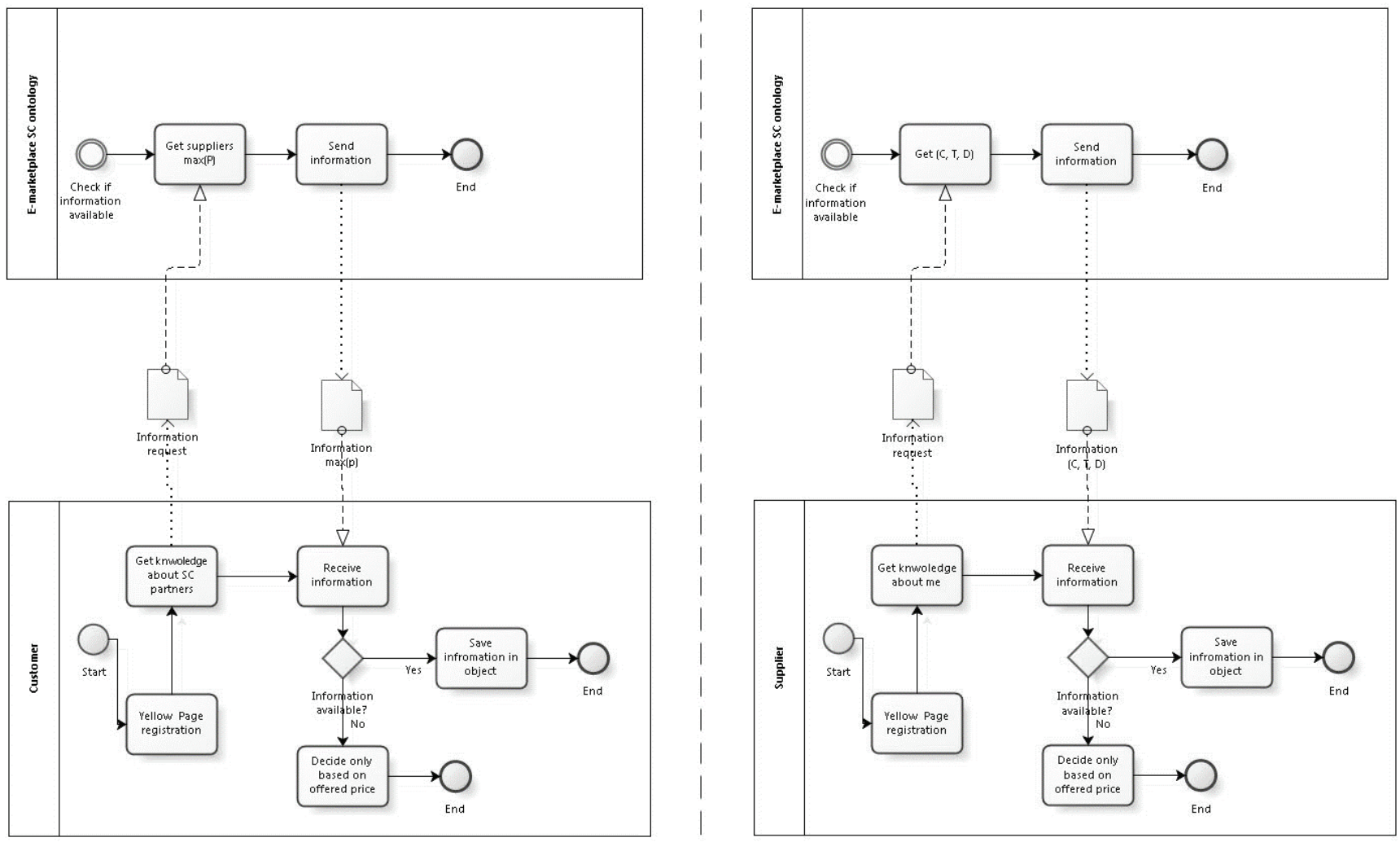

Figure 3 Registering (customer on left, supplier on right) in yellow page services and querying e-marketplace supply chain ontology

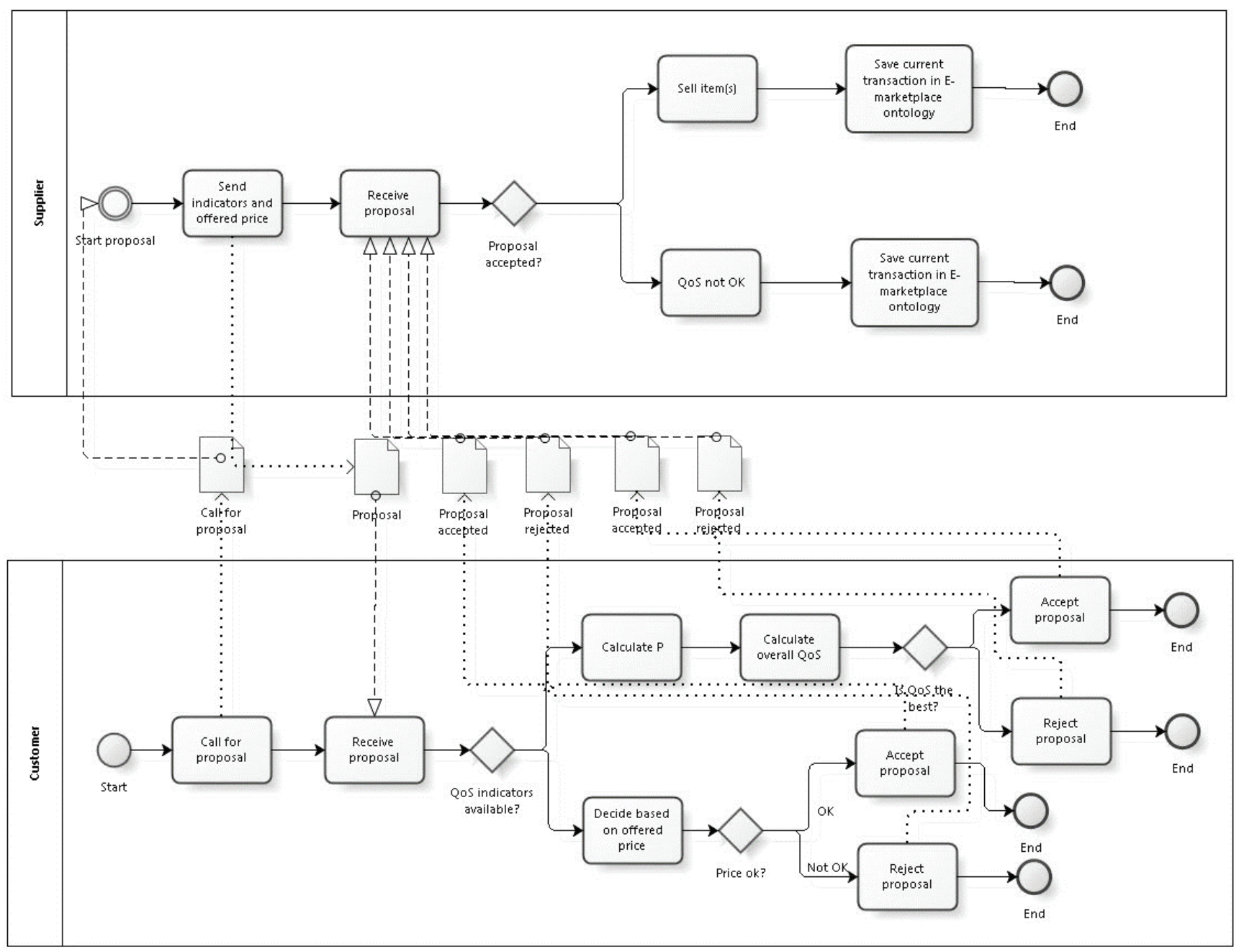

Figure 4 Supply chain agent behaviour 
When an agent of a type customer is being registered in yellow page services, the query to the e-marketplace ontology is made for all eligible suppliers in the supply chain. Based on that query, customer-related indicators are saved into CustomerKnowledge object, which consists of the following information:

- Agent name: unique identifier of supplier;

- Maximum price: maximum price from all suppliers' business transactions.

When a specific proposal is made by a supplier, a serialised object SupplierContentMessage, is contained in a supplier's message content. Based on that knowledge from suppliers (average values of: $C, T, D, \mathrm{Q}_{\mathrm{p}}$ indicators) and knowledge about a specific supplier (stored in CustomerKnowledge object) that is already known to the customer (offered price $(P)$ and maximum price $(\max [P])$, the overall price indicator is calculated based on Eq. (7). As all indicators are now known to a customer, a service quality of each supplier may be calculated next, based on Eq. (8).

\subsection{Agent Behaviour}

Let us consider service quality assessment in a typical ordering operational cycle:

(1) A call for proposals for an item is posted to all known suppliers; it contains the quantity of items we wish to order and the date of order fulfilment.

(2) Suppliers either propose their offers and provide the data on the price for the requested (amount of) items or refuse the request.

(3) The customer makes the decision on the acceptance of an individual offer based on the prices or (in our case) the service quality indicators (Q) of all suppliers who provided positive feedback to our inquiry.

(4) An order is made to the selected supplier by posting it an accept-proposal, while a reject-proposal is sent to all other participating suppliers.

(5) The order is either acknowledged as being fulfilled or a failure to fulfil the order is issued by the chosen supplier.

(6) Based on the outcome of the inquiry, service quality indicators of the supplier-nodes are recalculated to be considered in the next ordering operation.

In our order-cycle the supplier and customer nodes take over the initiator and participant roles respectively in the autonomous agents' communication according to the FIPA Contract Net Protocol. The mentioned data is transferred among them with appropriately tagged messages. The service quality for any supply chain node is maintained by its autonomous agent within its knowledge base. Initially (since there is no previous service quality data), the decision on an order is made solely based on price. With any subsequent order being fulfilled, however, the service quality is recalculated and the proposal selection decisions are made in an increasingly informed manner.

The communication between different types of agents starts when a customer requests a quotation (call for proposal) for its desired item (shown in Figure 4). After calls for proposals are made, proposals are sent from eligible registered suppliers to the customer. Based on own knowledge about suppliers and indicators sent from suppliers, the service quality calculation is done and a decision is made accordingly. If a procurement decision for a supplier's proposal is positive, the supplier is notified about proposal acceptance, otherwise a proposal refusal is sent to the supplier. Finally, the current transaction is saved into the e-marketplace supply chain ontology of the supplier to be accounted for in its future proposal considerations.

\section{EVALUATION \\ 3.1 Synthetically}

As an example of a supply chain process, let us consider a customer agent's order for material. There are two distinct cases that are being observed here:

(1) The customer decides to buy an item from a supplier based only on the price of the item. Here, the supplier's agent with the lowest price is chosen - overall $Q$ is calculated solely based on $Q_{\mathrm{p}}$.

(2) The customer decides to buy an item from suppliers' agents based on the service quality they offer. Here, all service quality criteria, defined above, are taken into consideration - overall $Q$ is measured based on $C, D$, $T$ and $Q_{\mathrm{p}}$.

The first case is obvious. The minimum price tag is chosen as best $Q_{\mathrm{p}}$. For the purpose of comparison, the offered prices are divided with the maximum price in order to normalise the indicator.

In the second case, we need to consider the service quality of every supplier.

The correctness indicator represents the proportion of correctly fulfilled orders, based on the supplier's records. If the number of correctly fulfilled orders (c) by the supplier is 38 and the total number of orders $(d)$ is 47 , then the correctness indicator $(C)$ of the supplier is $81 \%(0.81)$.

The timeliness indicator represents the proportion of on-time completed orders. If the number of timely fulfilled orders $(t)$ to our supplier is 40 , based on the same number of orders $(d)$ the timeliness $(T)$ indicator of the supplier is $85 \%(0.85)$.

The dependability $(D)$ indicator is calculated based on two indicators:

- If the node was down (unable to serve our requests) in $81 \%$ of the cases, its availability is $19 \%(A=0.19)$.

- If the node was unable to deliver the ordered amount of items in $6 \%$ of the cases, its reliability is $94 \%(R=$ $0.94)$.

- The dependability indicator $(D)$ of the supplier is represented by the average value of its availability $(A)$ and reliability $(R)$, which in our case is $56 \%(D=0.56)$.

Finally, the price indicator for the item is calculated. If the cost of material $(C M)$ is EUR 61, the processing quantity $(P Q)$ is 550 , the cost per processing unit (cp) is EUR 52, the time to process (TS) is 9 hours, the time to prepare $(T P)$ is 2 hours and cost of product processing time $(c t)$ is EUR 58 /hour, then the price of the supplier's item is EUR 29299 divided by 550, i.e. EUR 53.27. The price indicator again is calculated by dividing the individual prices, given by our suppliers, with the maximum price. 
With all the service quality indicators known, we can calculate the overall $Q$ indicator of the supplier that renders 0.72, considering the price difference between the maximum price (161.42) and supplier's price, which renders 0.65 as our $Q_{\mathrm{p}}$ indicator.

Taking the two cases into consideration, our conclusion is the following. If the customer decides to buy the item from the supplier based solely on price where he was the only bidder, the $Q$ would be $1-53.27 / 53.27=0$. In case there were two and the price tag of the other supplier was 161.42 , its $Q$ would be $1-53.27 / 161.42=$ 0.65 , whereas the other supplier's $Q$ would be 0 .

In contrast, if all the service quality criteria were taken into consideration then the $Q$ of the first supplier would be 0.72 . If we knew nothing about the other supplier, then obviously he would win, however if the second supplier was best in its field (with its other service quality indicators close to 1 ), his $Q$ would be 0.75 or higher and hence the second supplier would be chosen, despite the higher price tag.

Hence, by taking $C, T$, and $D$ indicators into consideration, the customer makes an informed decision based on the information on how his potential suppliers have been performing in the past, i.e. how many times:

- the correct product has been shipped to a customer,

- the delivery has been on time,

- supplier has been available and reliable in fulfilling customer's orders.

If the decision on the purchase were made solely based on the price of the product, the customer's risk of getting the wrong item or getting the right item too late would be much higher and could lead to dissatisfaction of his customers and consequently to lower overall service quality within his supply chain.

\subsection{Analytically}

In the second case, our model is evaluated based on 100 randomly generated samples of service quality entries with the calculated service quality indicators. Here, we consider random orders within our supply chain. The results are calculated based on our service quality assessment model and presented graphically.

After a specific supply chain transaction between any pair of observed supply chain nodes is processed, service quality is recalculated for a specific supply chain node involved in the transaction, and any future decisions are made based on the highest value of service quality indicator

The parameters of our model are generated randomly, since they cannot be gathered otherwise, i.e. without measuring them on a concrete supply chain:

- total number of orders $(d)$, distributed uniformly on the interval $(1: 1000)$

- number of correctly fulfilled orders $(c)$, distributed uniformly on the interval $(1: d)$

- number of timely fulfilled orders $(t)$, distributed uniformly on the interval $(1: d)$

- mean time to failure $(M T T F)$ in hours, distributed uniformly on the interval $(1: 8760)$

- mean time to repair $(M T T R)$ in hours, distributed uniformly on the interval $(1: M T T F)$

- ratio of failures $(F)$, distributed uniformly on the interval $(0,1)$

- cost of material $(C M)$, distributed uniformly on the interval $(1,100)$

- $\quad$ processing quantity $(P Q)$, distributed uniformly on the interval $(1: 1000)$

- $\quad$ cost per processing unit ( $c p)$, distributed uniformly on the interval $(1,100)$

- time to process/service time $(T S)$ in hours, distributed uniformly on the interval $(1: 24)$

- time to prepare $(T P)$ in hours, distributed uniformly on the interval $(1: 12)$

- with the appropriate cost of product/service processing time $(c t)$, distributed uniformly on the interval $(1,100)$

As basis for our analytical evaluation, a fiscal year with 8760 hours and 24-hour/day work schedules has been chosen. Since we cannot make any assumptions on the number of orders, we have chosen a random number from the $(1: 1000)$ interval for this indicator. Equally a random number from the $(0,1)$ interval has been chosen as the node's failure rate. To calculate the price, we have chosen the pertaining indicators to be random numbers from the stated intervals.

According to our test sample (cp. Tab. 1) in Fig. 5 one can observe the effects of the total $(d)$ and number of correctly fulfilled orders $(c)$ on the correctness indicator (C). The higher $c$ is, the higher is the overall $C$ on the $(0,1)$ interval.

Table 1 An example excerpt from our test sample

\begin{tabular}{|c|c|c|c|c|c|c|c|c|c|c|}
\hline$\#$ & $C$ & $T$ & $D$ & $A$ & $R$ & $P$ & $Q_{\text {p }}$ & $Q_{\max }$ \\
\hline 1 & 0,23 & 0,60 & 0,75 & 0,73 & 0,78 & 57,52 & 0,55 & 0,63 & 0,41 \\
\hline 2 & 0,75 & 0,25 & 0,61 & 0,58 & 0,64 & 79,65 & 0,53 & 0,49 & 0,91 \\
\hline 3 & 0,90 & 0,24 & 0,91 & 0,91 & 0,92 & 3,00 & 0,76 & 0,98 & 0,41 & 0,87 \\
\hline 4 & 0,38 & 0,40 & 0,75 & 0,70 & 0,79 & 38,93 & 0,57 & 0,75 & 0,41 & 0,94 \\
\hline 5 & 0,39 & 0,92 & 0,26 & 0,02 & 0,50 & 98,42 & 0,48 & 0,37 & 0,41 & 0,84 \\
\hline 6 & 0,55 & 0,97 & 0,63 & 0,50 & 0,76 & 68,43 & 0,68 & 0,56 & 0,41 & 0,89 \\
\hline 7 & 0,16 & 0,61 & 0,26 & 0,25 & 0,27 & 58,81 & 0,41 & 0,62 & 0,41 & 0,90 \\
\hline 8 & 0,32 & 0,15 & 0,41 & 0,18 & 0,63 & 47,63 & 0,39 & 0,70 & 0,41 & 0,92 \\
\hline 9 & 0,47 & 0,90 & 0,88 & 0,86 & 0,90 & 90,52 & 0,67 & 0,42 & 0,41 & 0,85 \\
\hline 10 & 0,44 & 0,84 & 0,72 & 0,58 & 0,85 & 51,88 & 0,67 & 0,67 & 0,41 & 0,91 \\
\hline
\end{tabular}

According to test samples (cp. Tab. 1) in Fig. 6 one can observe the effects of the total $(d)$ and the number of timely fulfilled orders $(t)$ on the timeliness indicator $(T)$. The higher $t$ is, the higher is the overall $T$ on the $(0,1)$ interval.
According to test samples (cp. Tab. 1) in Fig. 7 one can observe the effects of the mean times to fail and repair $(M T T F, M T T R)$ and the fault rate $(F)$ parameters on availability $(A)$ and reliability $(R)$ - their values are high as far as the MTTR/MTTF ratio and $F$ are low. The 
dependability $(D)$ indicator adequately summarises its components ( $A$ and $R$ ) according to the fact that both of them are considered equally important.

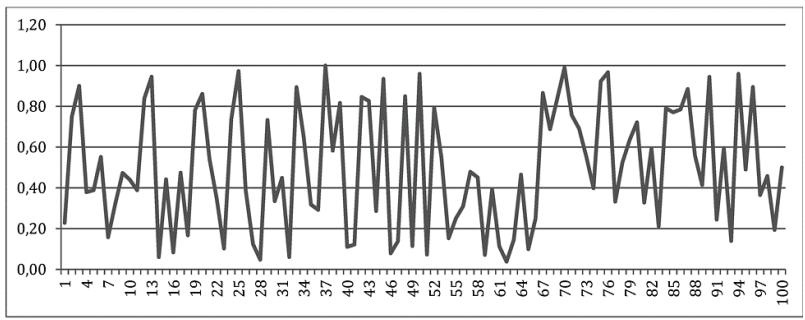

Figure 5 Correctness according to test samples

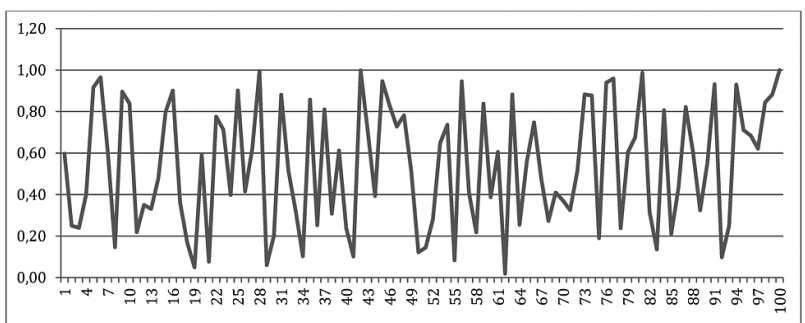

Figure 6 Timeliness according to test samples

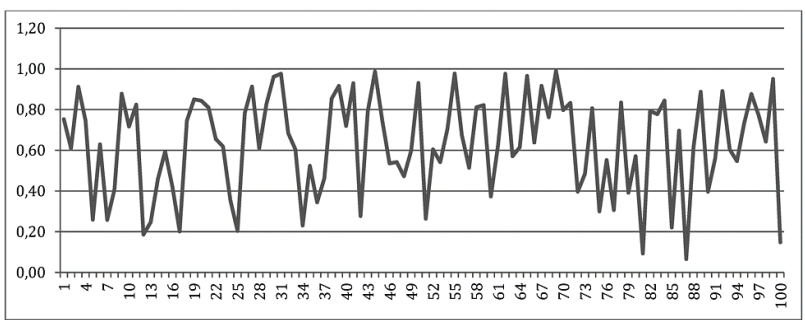

Figure 7 Dependability according to test samples

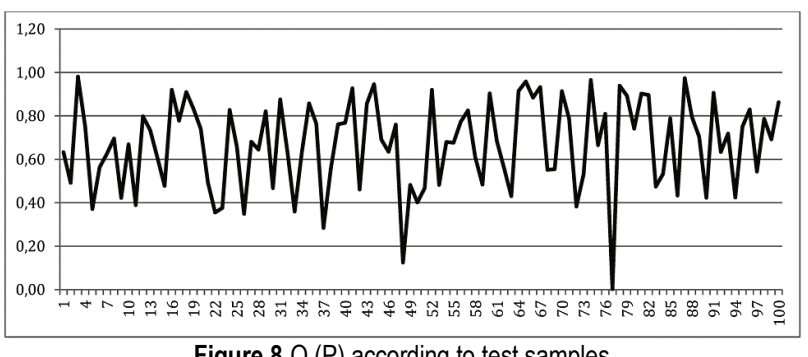

Figure $8 \mathrm{Q}(\mathrm{P})$ according to test samples

According to test samples (cp. Tab. 1) in Fig. 8 one can observe the implications of material price $(C M)$ and the individual costs of labour ( $T S, T P$ and $P Q$ ) on the overall price for an item. The price indicator $(P)$ is subsequently normalised by dividing it with its maximum value, since otherwise it would outvote other parameters in the composite service quality calculation.

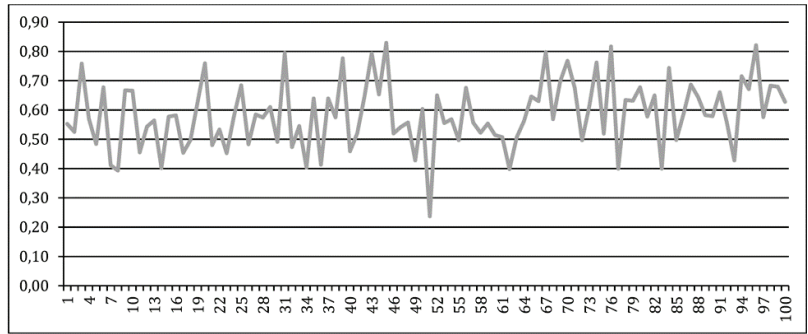

Figure $9 Q(C, T, D, P)$ according to test samples

According to test samples (cp. Tab. 1) in Fig. 9 the overall service quality indicator values (including all service quality criteria) are given. One can observe that the individual indicators $(C, T, D$ and $P)$ are truly independent from each other. Also evident is that overall quality $(Q)$ adequately summarises the given / calculated correctness $(C)$, timeliness $(T)$, dependability $(D)$ and price $(P)$ indicators, as defined in our analytical model.

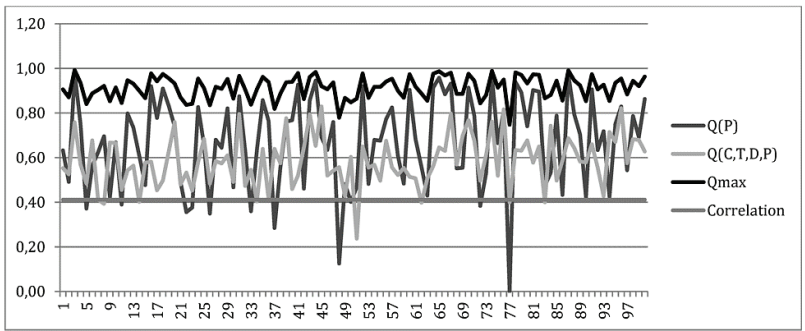

Figure $10 Q(C, T, D, P)$ vs. $Q(P)$

In Fig. 10 a comparison between overall quality $Q(P)$ based just on price and composite overall quality $Q(C, T$, $D, P)$ is outlined. One can observe that the composite quality values better resemble the optimum $Q_{\max }$ curve being based on maximum $C, T, D$ and current $P$ values and their oscillations are smaller as opposed to the $Q(P)$ curve, which still resembles the optimum curve, however its oscillations are much higher.

From the correlation among $Q(P)$ and $Q(C, T, D, P)$ one can see that $60 \%$ of purchasing decision information originates from non-price related indicators. This indicator varies a bit among test scenarios, however in general one may say that our purchasing decision based solely on price, would be suboptimal in about $50 \%$ of cases.

From our analysis we can conclude that the $Q(C, T, D$, $P$ ) values better resemble supply chain performance of the observed supply chain. Considering the individual values of $Q(C, T, D, P)$ and $Q(P)$, we can conclude that the decision model based on composite service quality evaluation would positively influence our business logic for two reasons:

(1) Composite service quality values prevent us from making quick uninformed decisions based solely on price variations.

(2) When considering the supply chain as a whole, the ordering process based on composite service quality evaluation would result in its lower oscillations. Since they usually represent some extraordinary events and offer us little information on their background, high oscillations in SCM are generally considered negative. In other words, for sustainable SCM lower service quality oscillations are better.

\section{CONCLUSION}

In supply chain management two approaches to service transformation in the digital era can be observed for efficient, service quality-oriented supply chain management:

(1) Centralised e-marketplace web portals, combining demand and supply in a temporally and spatially shared repository and

(2) Decentralised, agent-based e-marketplaces with a yellow-page dictionary service and distributed network of temporally and spatially distributed agents of supply chain partners. 
In our research the latter approach has been chosen, since it resembles the natural behaviour of supply chain nodes, which: appear, register their services, handle inquiries and orders and establish their own service quality-based supply chains on the supply chain network with their partners. In the article the structure, behaviour and communication models of the proposed multi-agent framework with a service quality oriented assessment model for collaboration within supply chains have been presented. Basic underlying e-commerce ontology has been introduced. The method for assessing the service quality and its adoption into supply chain management operations has been presented. As a conclusion, the service-quality-based supply chain management model has been statistically evaluated against randomly created ecommerce transactions. Our envisaged approach is to link a company's ERP order management system to our multiagent platform.

The most important finding of our research is that the service quality decision model adds more than $50 \%$ of information when choosing a supply chain partner for collaboration on the customer side (i.e. as opposed to decisions based solely on product/service price). Hence, we may conclude that by adopting our framework the service quality within supply chains can be significantly improved.

\section{REFERENCES}

[1] Zhang, W. \& Reimann, M. (2014). Towards a multiobjective performance assessment and optimization model of a two-echelon supply chain using SCOR metrics. Central European Journal of Operations Research, 22(4), 591-622. https://doi.org/10.1007/s10100-013-0294-7

[2] Niraj, R., Gupta, M., \& Narasimhan, C. (2001). Customer profitability in a supply chain. Journal of Marketing, 65(3), 1-16. https://doi.org/10.1509/jmkg.65.3.1.18332

[3] Ketchen, D. J., et al. (2008). Best value supply chains: A key competitive weapon for the $21^{\text {st }}$ century. Business Horizons, 51(3), 235-243. https://doi.org/10.1016/j.bushor.2008.01.012

[4] Seth, N., Deshmukh, S., \& Vrat, P. (2006). A framework for measurement of quality of service in supply chains. Supply Chain Management: An International Journal, 11(1), 82-94. https://doi.org/10.1108/13598540610642501

[5] Chow, G., Heaver, T. D., \& Henriksson, L. E. (1994). Logistics performance: definition and measurement. International journal of physical distribution \& logistics management, 24(1), 17-28. https://doi.org/10.1108/09600039410055981

[6] Sahay, B., et al. (2006). A conceptual model for quality of service in the supply chain. International Journal of Physical Distribution \& Logistics Management, 36(7), 547-575. https://doi.org/10.1108/09600030610684971

[7] Lyons, A., et al. (2012). Supply Chain Performance Measurement, in Customer-Driven Supply Chains. Springer London, 133-148. https://doi.org/10.1007/978-1-84628-876-0_7

[8] Mes, M., van der Heijden, M., \& Schuur, P. (2013). Interaction between intelligent agent strategies for real-time transportation planning. Central European Journal of Operations Research, 21(2), 337-358. https://doi.org/10.1007/s10100-011-0230-7

[9] Gumzej, R. \& Gajšek, B. (2013). Introducing quality of service criteria into supply chain management for excellence. Technological Solutions for Modern Logistics and Supply Chain Management, p. 70. https://doi.org/10.4018/978-1-4666-2773-4.ch006
[10] Gumzej, R., Sukjit, P., \& Unger, H. (2012). Modelling Overlay Networks for Autonomous Supply Chain Systems. Logistics \& Sustainable Transport, 3(2).

[11] Pal, K. \& Karakostas, B. (2014). A multi agent-based service framework for supply chain management. Procedia Computer Science, 32, 53-60. https://doi.org/10.1016/j.procs.2014.05.397

[12] Rashad, W. \& Gumzej, R. (2014). The Information Technology in Supply Chain Integration: Case Study of Reda Chemicals with Elemica. International Journal of Supply Chain Management, 3(1).

[13] Kim, K., Paulson Jr, B. C.; \& Petrie Jr, C. J. (1999). Agent based electronic markets for project supply chain coordination. Proceedings of the AAAI-00 Workshop on Knowledge-Based Electronic Markets, USA.

[14] Hilletofth, P., et al. (2016). Developing service supply chains by using agent based simulation. Industrial Management \& Data Systems, 116(2), 255-270. https://doi.org/10.1108//MDS-05-2015-0220

[15] Kovalchuk, Y. (2009). A Multi-agent decision support system for supply chain management, Citeseer.

[16] Rady, H. A. (2011). Multi-agent system for negotiation in a collaborative supply chain management. International Journal of Video \& Image Processing and Network Security IJVIPNS-IJENS, 11(5).

[17] Bearzotti, L. A., Salomone, E., \& Chiotti, O. J. (2012). An autonomous multi-agent approach to supply chain event management. International Journal of Production Economics, 135(1), 468-478. https://doi.org/10.1016/j.jpe.2011.08.023

[18] Ameri, F. \& McArthur, C. (2013). A multi-agent system for autonomous supply chain configuration. The International Journal of Advanced Manufacturing Technology, 66(5-8), 1097-1112. https://doi.org/10.1007/s00170-012-4392-9

[19] Domínguez, R., Cannella, S., \& Framinan, J. M. (2015). SCOPE: A Multi-Agent system tool for supply chain network analysis. EUROCON 2015 - International Conference on Computer as a Tool, IEEE. https://doi.org/10.1109/EUROCON.2015.7313688

[20] Dominguez, R., et al. (2013). Using multi-agent systems to explore information sharing in arborescent supply chain networks. Industrial Engineering and Systems Management (IESM), Proceedings of 2013 International Conference on. IEEE.

[21] Wang, G., Wong, T., \& Wang, X. (2013). An ontology based approach to organize multi-agent assisted supply chain negotiations. Computers \& Industrial Engineering, 65(1), 215. https://doi.org/10.1016/j.cie.2012.06.018

[22] Hernández, J. E., et al. (2014). Collaborative planning in multi-tier supply chains supported by a negotiation-based mechanism and multi-agent system. Group Decision and Negotiation, 23(2), 235-269. https://doi.org/10.1007/s10726-013-9358-2

[23] Sitek, P., Nielsen, I. E., \& Wikarek, J. (2014). A hybrid multi-agent approach to the solving supply chain problems. Procedia Computer Science, 35, 1557-1566. https://doi.org/10.1016/j.procs.2014.08.239

[24] Fu, J. \& Fu, Y. (2015). An adaptive multi-agent system for cost collaborative management in supply chains. Engineering Applications of Artificial Intelligence, 44, 91100. https://doi.org/10.1016/j.engappai.2015.05.002

[25] Kumari, S., et al. (2015). A multi-agent architecture for outsourcing SMEs manufacturing supply chain. Robotics and Computer-Integrated Manufacturing, 36, 36-44. https://doi.org/10.1016/.r.rim.2014.12.009

[26] Giannakis, M. \& Louis, M. (2011). A multi-agent based framework for supply chain risk management. Journal of Purchasing and Supply Management, 17(1), 23-31. https://doi.org/10.1016/j.pursup.2010.05.001 
[27] Bellifemine, F. L., Caire, G., \& Greenwood, D. (2007). Developing multi-agent systems with JADE. Vol. 7, John Wiley \& Sons. https://doi.org/10.1002/9780470058411

[28] Russell, S., Norvig, P., \& Intelligence, A. (1995). A modern approach. Artificial Intelligence. Prentice-Hall, Egnlewood Cliffs, 25, 27.

[29] Fensel, D. (2001). Ontologies, in Ontologies. Springer Berlin Heidelberg, 11-18. https://doi.org/10.1007/978-3-662-04396-7_2

[30] Bellifemine, F., Poggi, A., \& Rimassa, G. (2001). Developing multi-agent systems with a FIPA-compliant agent framework. Software-Practice and Experience, 31(2), 103-128.

https://doi.org/10.1002/1097-024X(200102)31:2<103::AID-

SPE358>3.0.CO;2-0

\section{Contact information:}

\section{Bojan MILIĆ}

Evolutia d.0.0.

Zabukovica 128d, 3302 Griže, Slovenia

Bojan ROSI, PhD, Full Professor

University of Maribor, Faculty of Logistics,

Mariborska cesta 7, 3000 Celje, Slovenia

E-mail: bojan.rosi@um.si

Roman GUMZEJ, PhD, Associate Professor University of Maribor, Faculty of Logistics,

Mariborska cesta 7, 3000 Celje, Slovenia

E-mail: roman.gumzej@um.si 